\section{Giant Cell Arteritis Revealed by Vertebrobasilar Insufficiency}

\section{To the Editor:}

Giant cell arteritis (GCA) is a rare cause of stroke involving cervical vertebral arteries. We describe a patient with no clinical symptoms of GCA and
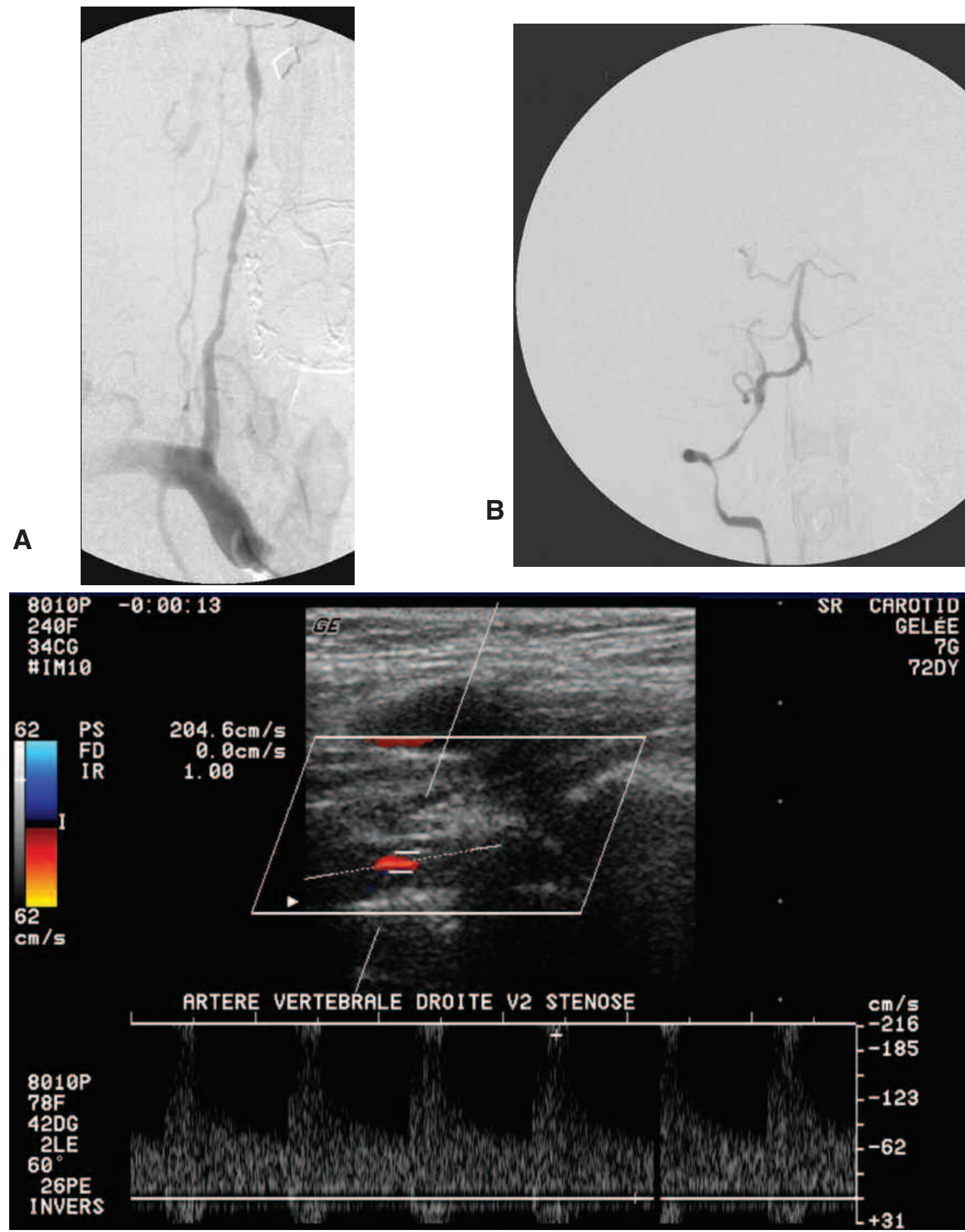

C

Figure 1. A. Right vertebral angiogram revealed multiple and concentric stenosis. B. Cerebral angiography revealed multiple stenosis of the right vertebral artery in the extradural portion, a normal diameter of the intradural portion and of the basilar artery, and occlusion of the left vertebral artery. C. Ultrasonography showed concentric stenosis of the right vertebral artery. several cardiovascular risk factors. GCA was considered because of multiple and concentric stenosis of the right cervical vertebral artery and occlusion of the left one.

A 76-year-old man was admitted with a 1-month history of progressive onset of right facial paresthesia, dysarthria, gait ataxia, vertigo, and left

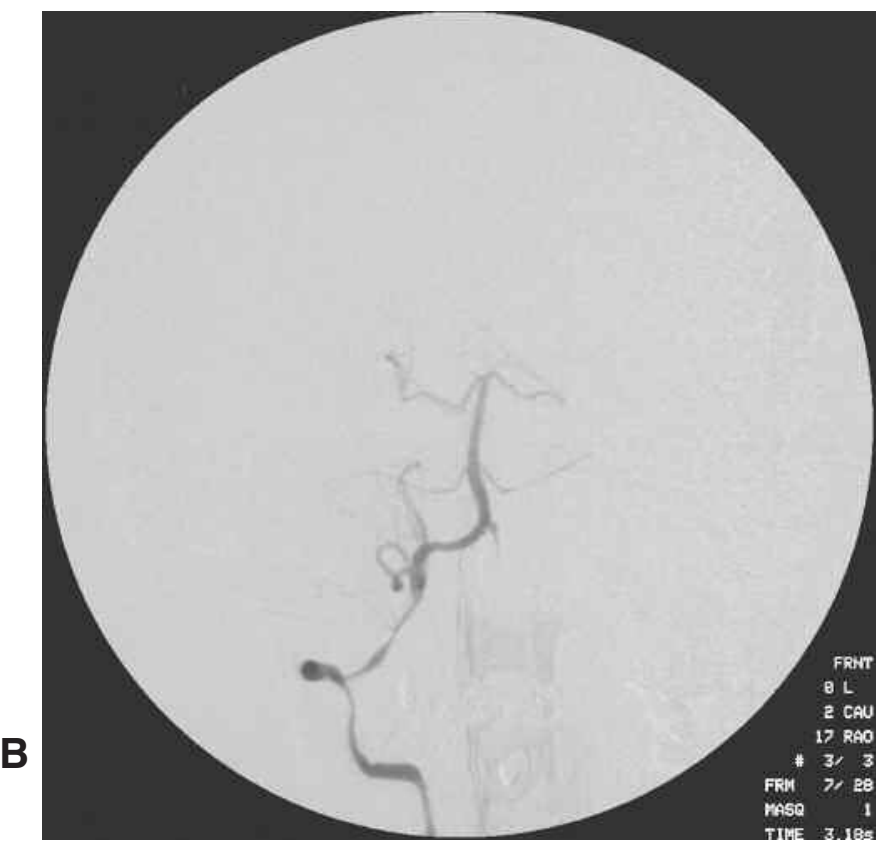


hemiparesis. He had a history of hypertension, hypercholesterolemia, smoking, and coronary disease, and was taking a daily dose of aspirin 75 mg. He did not complain of headache, and temporal artery palpation was normal. Brain magnetic resonance imaging (MRI) scan showed right pontine and left cerebellar infarctions. Cervical MR angiography revealed occlusion of the left vertebral artery and severe stenosis of the right. This was confirmed by cerebral angiography (Figure 1A, 1B). Ultrasonography (Figure 1C) showed a concentric aspect to the stenosis. Erythrocyte sedimentation rate (ESR) was $60 \mathrm{~mm} /$ hour.

The diagnosis of GCA was considered because of the patient's age, the aspect of the stenosis of the right vertebral artery, and the elevated ESR. The diagnosis was confirmed by temporal artery biopsy (TAB) showing inflammatory cell infiltrate, multinucleate giant cells, and intimal proliferation, with a complete luminal occlusion of the artery (Figure 2). Prednisone $70 \mathrm{mg} /$ day $(1 \mathrm{mg} / \mathrm{kg}$ ) was started and the daily dose of aspirin was increased to $160 \mathrm{mg}$. One month later, he complained of worsening dysarthria and ataxia. MRI showed new right pontine and left thalamic infarctions with a bilateral vertebral occlusion. He was given high-dose intravenous (IV) methylprednisolone (360 mg/day), and aspirin was replaced with heparin for activated partial thromboplastin time $>2$. His condition deteriorated, with ataxia and coma, and he died a few days later.

GCA causes vasculitis characterized by granulomatous inflammation in the wall of medium-size and large arteries and preferentially affects extracranial branches of the carotid artery ${ }^{1}$. Occlusion of the posterior ciliary arteries leading to anterior ischemic optic neuropathy is the most frequent complication, but cerebral arteries can also be involved, leading to cerebral infarction. Nesher, $e t a l^{2}$ reported cranial ischemic complications (including visual loss and stroke) in $25 \%$ of a group of patients with GCA, but Wiszniewska, et al $^{3}$ found only $0.15 \%$ of stroke in a population of 4086 patients with GCA. Vertebral arteries are more likely affected than internal carotid arteries, especially in the extradural portion, where there is more elastic tissue ${ }^{4}$. Our patient's cerebral angiography showed multiple stenosis of this portion of the right vertebral artery, but the intracranial portion and the basilar artery were of normal diameter without stenosis (Figure 1B). Involvement of intracranial arteries is rare ${ }^{4}$, and cerebral infarctions are the hemodynamic consequences of occlusion of cervical arteries.

The ultrasonographic axial plan can show a concentric halo and T2weigted MRI a slightly hyperintense vessel wall due to inflammatory mural thickening of arteries 5 .

The prognosis of GCA of the vertebral arteries is poor: reports reveal that most patients die or become dependent ${ }^{6}$. Sutter, et al ${ }^{7}$ and Ahdab, et $a l^{8}$ described cases with good outcome. The patients received high-dose IV

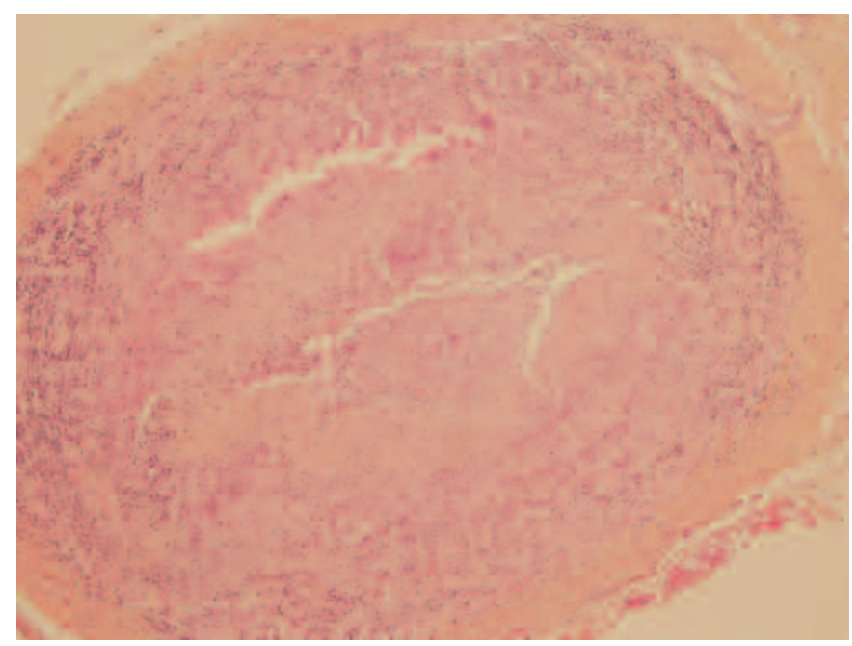

Figure 2. Temporal artery biopsy (HES obj*10) showed inflammatory cell infiltrate with intimal proliferation with a complete luminal occlusion. This image $=60 \%$ of original magnification . methylprednisolone (500 to $1000 \mathrm{mg} /$ day) for 5 days. Addition of other immunosuppressive treatments like cyclophosphamide is discussed, but no guidelines are put forward. Nesher, et $a l^{2}$ and Lee, et $a l^{9}$ suggested that antiplatelet or anticoagulation therapy may reduce ischemic complications.

Our patient had no symptom of GCA, with no headache, but the characteristics of multiple and concentric stenosis of the right vertebral artery pointed toward the diagnosis. TAB not only confirmed the diagnosis but also revealed a complete luminal obstruction. Makkuni, et al ${ }^{10}$ suggested that the degree of intimal hyperplasia was associated with ischemic complications in GCA.

Even if clinical symptoms are not evident, GCA should be considered in patients over age 50 years with multiple and concentric stenosis of extradural vertebral arteries. Diagnosis may be difficult in the absence of headache and presence of atherosclerotic risk factors, but poor prognosis can be worsened by the delay of immunosuppressive treatment.

SÉBASTIEN RICHARD, MD, Service de Neurologie; RENÉ ANXIONNAT, PhD, Service de Neuroradiologie Diagnostique et Thérapeutique; CAMILLE DELAUNAY, MD, Service Anatomie et Cytologie Pathologiques; XAVIER DUCROCQ, PhD, CHU Nancy, Hopital Central, 29 avenue du Marechal de Lattre de Tassigny-CO 34, 54035 Nancy Cedex, France. Address reprint requests to Dr. Richard; E-mail: s.richard@chu-nancy.fr

\section{REFERENCES}

1. Warrington KJ, Matteson EL. Management guidelines and outcome measures in giant cell arteritis. Clin Exp Rheumatol 2007;25:137-41.

2. Nesher G, Berkun Y, Mates M, Baras M, Rubinow A, Sonnenblick $\mathrm{M}$. Low-dose aspirin and prevention of cranial ischemic complications in giant cell arteritis. Arthritis Rheum 2004; 50:1332-7.

3. Wiszniewska M, Devuyst G, Bogousslavsky J. Giant cell arteritis as a cause of first-ever stroke. Cerebrovasc Dis 2007;24:226-30.

4. Salvarani C, Giannini C, Miller DV, Hunder G. Giant cell arteritis: involvement of intracranial arteries. Arthritis Rheum 2006; 55:985-9.

5. Reinhard M, Schmidt D, Schumacher M, Hetzel A. Involvement of the vertebral arteries in giant cell arteritis mimicking vertebral dissection. J Neurol 2003;250:1006-9.

6. Ruegg S, Engelter S, Jeanneret C, et al. Bilateral vertebral artery occlusion resulting from giant cell arteritis: report of 3 cases and review of the literature. Medicine 2003;82:1-12.

7. Sutter R, Renaud S, Bonati L, et al. Bilateral vertebral giant cell arteritis - favourable outcome in two cases. J Neurol 2008;255:133-4.

8. Ahdab R, Thabuy F, Menager de Froberville E, Brugieres P, Hosseini H. Reversible vertebral artery stenosis following corticotherapy in giant cell arteritis. Eur Neurol 2008;59:331.

9. Lee MS, Smith SD, Galor A, Hoffman GS. Antiplatelet and anticoagulant therapy in patients with giant cell arteritis. Arthritis Rheum 2006;54:3306-9.

10. Makkuni D, Bharadwaj A, Wolfe K, Payne S, Hutchings A, Dasgupta B. Is intimal hyperplasia a marker of neuro-ophthalmic complications of giant cell arteritis? Rheumatology Oxford 2008;47:488-90.

J Rheumatol 2009;36:2; doi:10.3899/jrheum.080922 